\title{
Diffusion Dynamics in Economics: an Application to the Effects of Fiscal Policy
}

\author{
Orlando Gomes ${ }^{1}, 2$
}

- Version 2, July 2013 -

${ }^{1}$ Instituto Superior de Contabilidade e Administração de Lisboa (ISCAL) and Business Research Unit [BRU/UNIDE/ISCTE]. E-mail: omgomes@iscal.ipl.pt. Address: Instituto Superior de Contabilidade e Administração de Lisboa (ISCAL), Av. Miguel Bombarda 20, 1069-035 Lisbon, Portugal.

${ }^{2} \mathrm{~A}$ previous version of this paper was presented in the $24^{\text {th }}$ European Conference on Operations Research (Euro XXIV) [Lisbon University, July 2010]. I would like to thank the organizers and the participants. A special thanks goes to my colleague Ana Cristina Lino Marques. I also acknowlege the helpful comments of Alan John Guimarães on the current version of the manuscript. The usual disclaimer applies. 


\begin{abstract}
This study addresses diffusion dynamics in an economic environment in which fiscal policy changes take place. The discussion, based upon a conventional intertemporal optimization setup, involves the consideration of a peculiar form of bounded rationality: it is assumed that only a small share of households is able to instantly recompute the optimal solution once the value of a tax rate is disturbed; all the other agents will then, gradually, follow the behavior of the first group (this can occur through contagion, social influence or social learning). As a result, the convergence towards the post-perturbation steady-state tends to follow a diffusion process and, consequently, policy measures may take time in affecting pervasively labor-leisure and consumptionsavings choices.
\end{abstract}

Keywords: Fiscal policy, Diffusion processes, Utility maximization, Laborleisure choices. 
Diffusion Dynamics in Economics: an Application to Fiscal Policy

\section{Introduction}

Mainstream macroeconomic analysis typically adopts a rationality concept according to which each agent is endowed with an automatic capacity to maximize intertemporal utility subject to some resource constraints. Recent work searches for reasonable departures from this paradigm of full rationality in order to better replicate observable phenomena. 'Bounded rationality', 'near-rational behavior / expectations' or 'limited rationality' are some of the designations that have been progressively introduced in the macroeconomic lexicon in order to characterize more or less significant departures from the idyllic setting in which every agent possesses the capabilities needed to compute optimal solutions in every circumstance and in an instantaneous way. The voluminous literature that touches the mentioned subjects includes the following relevant references: Akerlof and Yellen (1985), Haltiwanger and Waldman (1989), Bomfim and Diebold (1997), Beeby, Hall and Henry (2001), Weder (2004) and Gomes, Mendes and Mendes (2008).

With this study, we intend to contribute to the literature on bounded rationality in macroeconomics by imposing the following central assumption: only a small share of educated and well informed agents in the economy will be able or willing to solve the optimization problem they face (these can be called the 'innovators'). All the other agents will just imitate the behavior of the first group, but with a time lag that varies across individuals, following a diffusion process (these are the 'adopters' or 'imitators').

The general setting is a trivial intertemporal utility maximization problem where three types of taxes influence the choices of private agents: labor income taxes, taxes on asset revenues and taxes over the acquisition of consumption goods. The optimization problem is solved, allowing for presenting a trivial long-term outcome with constant values for the assumed endogenous variables (in the case, wealth, consumption and time allocation between labor and leisure). The benchmark setting for the analysis is this steady-state, which can be disturbed through changes in the way the fiscal policy is conducted. Any perturbation on one of the assumed tax rates will trigger a re-optimization process. According to the bounded rationality assumption, 
only a small share of agents (which in aggregate terms might correspond to an infinitesimal share) will instantly update their behavior and compute the new optimal solution; every other agent will follow in time according to some diffusion pattern.

The diffusion dynamics are adapted from Young (2009), who considers three categories of sources of diffusion: contagion, social influence and social learning. We will study the three, for different kinds of policy changes, in order to understand how consumption and labor-leisure choices are eventually affected on the aggregate. We will encounter inertia on the time paths of macro variables following the policy shocks: there is not an instantaneous jump from one equilibrium point to another one generated by the disturbance, but a gradual adjustment. I.e., after a tax change, the large majority of the agents (or even the entire population) will end up by adopting the same behavior as the individuals with the instantaneous re-optimization ability, however this will not occur for everyone at the same time; rather, a sluggish adjustment will be observed. Assuming some standard distribution of agents' attentiveness (e.g., a normal distribution) as we will do in the cases of social influence and social learning, or a simple logistic pattern, as in the contagion scenario, we will encounter a diffusion path for the evolution of the assumed endogenous variables or, which is the same, a hump-shaped evolution for the rate of change of the mentioned variables.

The central piece of the diffusion analysis will be the time allocation choice. The problem involves a state variable, wealth, that reacts immediately to tax changes, and two control variables, consumption and time allocation between labor and leisure. When a fiscal policy disturbance takes place, agents will take time (following the diffusion process) to apprehend such change in terms of their labor-leisure decision; as a result, some agents will continue, for some time length, to offer an amount of labor supply that is compatible with the pre-perturbation optimum but that is sub-optimal for the new tax rate. Consumption levels will be the direct outcome of financial and labor income, and therefore a non optimal labor-leisure choice will conduct to an amount of consumption that does not allow for maximizing the steady-state aggregate level of utility, as long as the diffusion process is not 
Diffusion Dynamics in Economics: an Application to Fiscal Policy

extinguished.

An important remark relates to the causes of sluggishness in the labor market: we are not departing from a perfectly competitive environment, i.e., the labor market is fully flexible. The causes for stickiness in the behavior of workers relate to their incapacity to immediately adjust to a change in the relevant parameters: only when observing the behavior of others, will the majority of the agents progressively adapt their decisions to the new economic conditions. In other words, it will be less costly for most of the agents to 'adopt' an existing behavior with some delay than to engage in a systematic re-optimization process (although we do not directly consider re-optimization costs, they will implicitly exist; they are the reason for the diffusion process to take place).

The remainder of the chapter is organized as follows: section 2 describes the structure of the model and section 3 characterizes the trivial steady-state of the problem. In section 4, we discuss types of diffusion processes, which are applied in sections 5 and 6 to address transitional dynamics under diffusion, after a policy shock. The first of these two sections concentrates on the impact of policy measures over the evolution of endogenous variables while the latter focuses its attention on long-run utility or welfare implications. Section 7 concludes.

\section{The Optimization Problem}

Consider an economy populated by a large number of individual households. They all face the same optimization problem, which consists in maximizing intertemporal utility, given an infinite horizon. The arguments of the instantaneous utility function are consumption, $c_{t}$, and the amount of time allocated to leisure. Normalizing the amount of available time to the unity, $\ell_{t} \in(0,1)$ will represent the share of time allocated to working hours, while $1-\ell_{t}$ will be the percentage of the household's time destined to leisure. Both variables, $c_{t}$ and $\ell_{t}$, are control variables for the agent, meaning that the problem faced by households consists in evaluating consumption - savings and labor - leisure trade-offs that best serve the goal of utility maximization. 
Diffusion Dynamics in Economics: an Application to Fiscal Policy

Let $\beta \in(0,1)$ represent the intertemporal discount factor. The individual agent maximizes the value of the following objective function:

$$
V_{0}=\sum_{t=0}^{\infty} \beta^{t} u\left(c_{t}, 1-\ell_{t}\right)
$$

Utility function $u(\cdot)$ is continuous, twice differentiable, increasing on its two arguments and subject to decreasing marginal returns, also relatively to both arguments, i.e., the following derivative signs must hold: $u_{c}>0, u_{c c}<0$ and $u_{1-\ell}>0, u_{1-\ell, 1-\ell}<0$. One also considers that the utility function is additively separable, that is, $u_{c, 1-\ell}=u_{1-\ell, c}=0$.

The optimization problem is subject to a resource constraint. This constraint reflects the accumulation of wealth. Households increase their wealth levels through labor participation, which pays a fixed wage rate, and through the accumulation of financial assets. In order to obtain a steady-state result such that wealth, consumption and labor and leisure shares are kept constant, we assume that assets' accumulation is subject to decreasing returns: additional returns on financial investments fall with the amount of wealth already applied in the markets (this view can be supported on the idea that more profitable investment opportunities are the first to be seized).

Three types of taxes will be considered in this economy: taxes over consumption, taxes over labor income and taxes over the returns on financial wealth. The corresponding rates are: $\tau_{c}, \tau_{\ell}, \tau_{s} \in(0,1)$. These rates will appear on the resource constraint, which takes the form

$$
a_{t+1}-a_{t}=\left(1-\tau_{\ell}\right) w \ell_{t}+\left(1-\tau_{s}\right) r a_{t}^{\alpha}-\left(1+\tau_{c}\right) c_{t}, a_{0} \text { given }
$$

In difference equation (2), $a_{t}$ represents the level of wealth held by the households at time $t, w$ translates a constant wage rate, $r$ is a parameter that allows to measure the returns on financial wealth and elasticity $\alpha \in$ $(0,1)$ reflects the degree of decreasing returns on the accumulation of wealth. Increasing consumption levels or assuming higher taxes (independently of their type) constitutes a penalty over the amount of available wealth (it will suffer a negative change). 
It is straightforward to solve the optimization problem $\operatorname{Max} V_{0}$ subject to resource constraint (2). The computation of first order conditions requires writing the Hamiltonian function,

$$
\begin{aligned}
H\left(a_{t}, c_{t}, \ell_{t}\right)= & u\left(c_{t}, 1-\ell_{t}\right)+ \\
& \beta p_{t+1}\left[\left(1-\tau_{\ell}\right) w \ell_{t}+\left(1-\tau_{s}\right) r a_{t}^{\alpha}-\left(1+\tau_{c}\right) c_{t}\right]
\end{aligned}
$$

with $p_{t}$ the co-state variable associated to the state variable $a_{t}$.

To derive optimality conditions we resort to the Pontryagin's principle; the necessary conditions for optimality are:

$$
\begin{gathered}
\frac{\partial H}{\partial c}=0 \Rightarrow u_{c}=\left(1+\tau_{c}\right) \beta p_{t+1} \\
\frac{\partial H}{\partial \ell}=0 \Rightarrow u_{\ell}=\left(1-\tau_{\ell}\right) w \beta p_{t+1} \\
\beta p_{t+1}-p_{t}=-\frac{\partial H}{\partial a_{t}} \Rightarrow\left[1+\alpha\left(1-\tau_{s}\right) r a_{t}^{-(1-\alpha)}\right] \beta p_{t+1}=p_{t}
\end{gathered}
$$

The transversality condition $\lim _{t \rightarrow \infty} p_{t} \beta^{t} a_{t}=0$ needs to be imposed in order to avoid the possibility of perpetual debt.

To develop further the optimality relations, we need to specify a functional form for the utility function. A shape that obeys to the properties one has imposed to this function is the following: $u\left(c_{t}, 1-\ell_{t}\right)=\ln \left[c_{t}\left(1-\ell_{t}\right)^{m}\right]$, where $m>0$ is the parameter reflecting the relevance that the household attributes to the utility of leisure relatively to consumption utility. For the chosen function, $u_{c}=1 / c_{t}$ and $u_{1-\ell}=m /\left(1-\ell_{t}\right)$.

Optimality conditions (4) and (6) allow for presenting an equation of motion for consumption,

$$
c_{t+1}=\beta\left[1+\alpha\left(1-\tau_{s}\right) r a_{t+1}^{-(1-\alpha)}\right] c_{t}
$$

By combining (4) and (5), one also encounters a static relation between 
share $\ell_{t}$ and the value of the consumption variable:

$$
\ell_{t}=1-\frac{m\left(1+\tau_{c}\right)}{w\left(1-\tau_{\ell}\right)} c_{t}
$$

As previously referred, the study to pursue is restricted to the evaluation of the steady-state. Thus, we will resort to equations (2), (7) and (8) to find the steady-state values $a^{*}:=a_{t+1}=a_{t}, c^{*}:=c_{t+1}=c_{t}$ and $\ell^{*}:=\ell_{t+1}=\ell_{t}$. Some algebra directs us to the intended expressions:

$$
\begin{gathered}
a^{*}=\left[\frac{\alpha \beta}{1-\beta}\left(1-\tau_{s}\right) r\right]^{1 /(1-\alpha)} \\
\ell^{*}=\frac{1}{1+m}\left\{1-\left(\frac{\alpha \beta}{1-\beta}\right)^{\alpha /(1-\alpha)} \frac{m\left[\left(1-\tau_{s}\right) r\right]^{1 /(1-\alpha)}}{w\left(1-\tau_{\ell}\right)}\right\} \\
c^{*}=\frac{1}{1+m}\left\{w \frac{1-\tau_{\ell}}{1+\tau_{c}}+\left(\frac{\alpha \beta}{1-\beta}\right)^{\alpha /(1-\alpha)} \frac{\left[\left(1-\tau_{s}\right) r\right]^{1 /(1-\alpha)}}{1+\tau_{c}}\right\}
\end{gathered}
$$

\section{Long-term Policy Implications}

Long-term effects of policy changes are straightforward to obtain from the steady-state results.

Proposition 1 Under the proposed setting, an increase on the tax rate on savings has the following long-run impact over the economy: wealth and consumption levels fall and the share of time allocated to working hours suffers a positive change.

Proof. Just compute first-order derivatives of (9), (10) and (11) with respect to $\tau_{s}$; we find the results that are evidenced in the proposition, i.e., $\frac{\partial a^{*}}{\partial \tau_{s}}<0, \frac{\partial c^{*}}{\partial \tau_{s}}<0$ and $\frac{\partial \ell^{*}}{\partial \tau_{s}}>0$

The presented result is intuitive. Whenever a fiscal policy change correspond to an increase on the tax rate over financial wealth earnings, this will lower the accumulated wealth and the resources the agent will have available 
Diffusion Dynamics in Economics: an Application to Fiscal Policy

to consume. If earnings on savings are lowered by a tax increase, then the agent will need to resort to other sources of income, namely she will exchange leisure time by labor time, i.e., share $\ell^{*}$ is positively influenced.

For the tax rate on labor income the following outcome is found,

Proposition 2 When the government increases the labor income tax rate, this does not have any impact on the level of wealth. Consumption and the labor share will suffer, in this case, a negative change.

Proof. Again, compute the derivatives of the steady-state expressions, now with respect to tax rate $\tau_{\ell}$; results are $\frac{\partial a^{*}}{\partial \tau_{\ell}}=0, \frac{\partial c^{*}}{\partial \tau_{\ell}}<0$ and $\frac{\partial \ell^{*}}{\partial \tau_{\ell}}<0$

Now, the intuition is as follows: by disturbing labor income with a higher tax rate, the willingness to allocate time to working hours falls, i.e., agents will renounce some labor time in favor of leisure time. This reallocation of labor will lead to a lower income that implies lower steady-state consumption levels.

Finally, one can analyze the effect of rising taxes over consumption. A larger tax rate $\tau_{c}$ has the impact that the following proposition describes.

Proposition 3 A positive change in the tax rate on consumption will not influence wealth or the choice concerning the allocation of the household's available time. Relatively to consumption, the long-term value of the variable falls.

Proof. Proceeding as in the previous occasions, one computes partial derivatives of steady-state values with respect to the tax rate under scrutiny to find the corresponding signs, $\frac{\partial a^{*}}{\partial \tau_{c}}=\frac{\partial \ell^{*}}{\partial \tau_{c}}=0$ and $\frac{\partial c^{*}}{\partial \tau_{c}}<0$

A larger tax rate on consumption has impact only over consumption itself. As one should expect, a tax on consumption lowers the amount of available resources that the agent will have available to consume.

Another point deserving evaluation relates to the steady-state level of utility or welfare. For the specified utility function, the steady-state level of 
utility will be:

$$
\begin{gathered}
U\left(c^{*}, 1-\ell^{*}\right)= \\
\ln \left(\frac{m^{m} w}{(1+m)^{1+m}} \frac{1-\tau_{\ell}}{1+\tau_{c}}\left\{1+\left(\frac{\alpha \beta}{1-\beta}\right)^{\frac{\alpha}{1-\alpha}} \frac{\left[\left(1-\tau_{s}\right) r\right]^{1 /(1-\alpha)}}{w\left(1-\tau_{\ell}\right)}\right\}^{1+m}\right)
\end{gathered}
$$

The evaluation of expression (12) allows us to understand the impact of fiscal policy changes over long-term utility.

Proposition 4 Long-run utility falls with an increase on savings or consumption tax rates. Utility can vary positively or negatively following a positive change on the labor income tax rate; the positive variation requires the following inequality to hold:

$$
\tau_{\ell}>1-\left(\frac{\alpha \beta}{1-\beta}\right)^{\frac{\alpha}{1-\alpha}} \frac{m}{w}\left[\left(1-\tau_{s}\right) r\right]^{1 /(1-\alpha)}
$$

Proof. The signs of the derivatives of steady-state utility with respect to $\tau_{s}$ and $\tau_{c}$ are straightforward to obtain from expression (12): $\frac{\partial U^{*}}{\partial \tau_{s}}<0$ and $\frac{\partial U^{*}}{\partial \tau_{c}}<0$. In what concerns the effect of the labor income tax over utility, the computation of the corresponding derivative gives place to

$$
\frac{\partial U^{*}}{\partial \tau_{\ell}}=\frac{1}{1-\tau_{\ell}}\left[(1+m) \frac{\phi}{1+\phi}-1\right]
$$

with $\phi:=\left(\frac{\alpha \beta}{1-\beta}\right)^{\frac{\alpha}{1-\alpha}} \frac{\left[\left(1-\tau_{s}\right) r\right]^{1 /(1-\alpha)}}{w\left(1-\tau_{\ell}\right)}$. The above derivative possesses a positive sign if $\phi>1 / m$. Rearranging, the inequality in the proposition is immediately found

To improve our understanding of the result in proposition 4 , we take a numerical example. The selected values are: $m=0.2, w=0.25, r=0.1$, $\alpha=0.25$ and $\beta=0.96$. In this case, $\tau_{\ell}=1-0.0675\left(1-\tau_{s}\right)^{1.3333}$ is the borderline between the two possible outcomes (increasing and decreasing utility following a positive change on $\tau_{\ell}$ ). For the specified values, for instance if $\tau_{s}=0, \tau_{s}=0.25$, or $\tau_{s}=0.5$ then a positive change in utility as the result of $\Delta \tau_{\ell}>0$ requires, respectively, $\tau_{\ell}>0.9325, \tau_{\ell}>0.954$, or $\tau_{\ell}>0.9732$. 
Diffusion Dynamics in Economics: an Application to Fiscal Policy

The information revealed by the example is that an extremely high tax rate on the labor income (relatively to the tax rate on savings) allows for an increase in steady-state utility whenever the tax rate on labor income incurs in a positive change. According to the numbers, though, the most likely result (the only admissible for empirically reasonable tax rates) is the opposite: long-run utility will fall with an increase in $\tau_{\ell}$. When the tax on labor earnings increases, the utility will increase through a direct effect (individuals exchange labor by leisure) and an indirect effect will take place as well (diverting labor hours towards leisure time implies lower income levels and therefore consumption will also decline, leading to a fall in utility). According to the proposed example, the second effect tends to dominate, and this becomes increasingly true as the other tax rate $\left(\tau_{s}\right)$ assumes larger values.

\section{A Typology of Diffusion Processes}

Based on Young (2009), we consider three classes of diffusion processes. Diffusion can occur through contagion, social influence or social learning. Each type of diffusion process has distinctive features, however a common trace exists: in every case, the variable subject to diffusion eventually follows an s-shaped evolution pattern.

Diffusion under contagion implies that people will adopt a given behavior just by being in contact with other individuals that have already adopted such behavior. Diffusion occurs, in this case, much as an epidemic. Models of diffusion by contagion were pioneered by Bass $(1969,1980)$ and can be synthesized in a simple logistic dynamic equation of the type:

$$
\Delta n_{t}=\left(\varphi_{I}^{c} n_{t}+\varphi_{E}^{c}\right)\left(1-n_{t}\right), \quad n_{0} \text { given }
$$

Variable $n_{t}$ denotes the share of individual agents that have already accessed a given new set of information or that have already adopted a certain behavior; $\Delta n_{t}:=n_{t+1}-n_{t}$ represents the time change of the assumed endogenous variable. Parameters $\varphi_{I}^{c}$ and $\varphi_{E}^{c}$ are both positive values and they translate the rates at which a current non-adopter 'hears about' the inno- 
vation from, respectively, previous adopters and from some external source [in equation (13), this idea is reflected on the fact that $\varphi_{I}^{c}$ is associated with $n_{t}$ while parameter $\varphi_{E}^{c}$ is not]. In the extreme case where $\varphi_{E}^{c}=0$, i.e., when contagion is only due to internal sources, we will be in the presence of a conventional s-shaped logistic equation and $\varphi_{I}^{c}$ will represent the rate of contagion (thus, it should be a value lower than 1 ).

To study the main properties of the contagion equation (13), we begin by computing the steady-state value of $n_{t}$. Letting $n^{*}:=n_{t+1}=n_{t}$ we find two solutions: $n^{*}=-\varphi_{E}^{c} / \varphi_{I}^{c}$ and $n^{*}=1$; only the second solution is admissible, because the parameters are both positive values and the share $n_{t}$ must lie, at every time moment, between 0 and 1 . Thus, the contagion effect implies an equilibrium result where all the universe of individual agents has adopted the behavior or has taken contact with the relevant new information. Furthermore, it is straightforward to realize that the steady-state is a stable point for any $n_{0} \in[0,1]$; to accomplish this result, we just need to notice that, according to (13), $\Delta n_{t} \geq 0$ for all possible values of $n_{t}$, i.e., share $n_{t}$ will increase in time from any admissible initial state until point $n^{*}=1$ is reached. Given this global stability result, we can consider a complete diffusion process, that is, we can start at $n_{0}=0$.

The most relevant property concerning equation (13) is presented in proposition 5 .

Proposition 5 Diffusion by contagion allows for an s-shaped adoption process as long as $\varphi_{I}^{c}>\varphi_{E}^{c}$. The acceleration phase (the phase in which the adoption curve is convex) cannot go beyond the fifty percent adoption level.

Proof. Consider the relation between $n_{t}$ and $\Delta n_{t}$. One has already verified that if $n_{t}=1$ then $\Delta n_{t}=0$; this is the steady-state point. We can also compute the instantaneous change in the value of $n_{t}$ when this share is equal to zero; replacing $n_{t}$ by zero into (13) we obtain $\Delta n_{t}=\varphi_{E}^{c}$. The diffusion process will display an s-shaped form if, starting at $n_{t}=0$, the adoption path is, in a first phase, convex and then, following an inflection point, it becomes concave. This inflection point in the time path of $n_{t}$ translates into a maximum for the function $\Delta n_{t}=f\left(n_{t}\right)$. If we find a maximum for this 
Diffusion Dynamics in Economics: an Application to Fiscal Policy

function, in the interval $n_{t} \in[0,1]$, then the s-shaped diffusion process is confirmed.

The necessary conditions for the existence of a maximum of any twice continuously differentiable function $f\left(n_{t}\right)$ are $f^{\prime}=0$ and $f^{\prime \prime} \leq 0$. In the case under appreciation, $f^{\prime}=\varphi_{I}^{c}\left(1-2 n_{t}\right)-\varphi_{E}^{c}$ and $f^{\prime \prime}=-2 \varphi_{I}^{c}$. The secondorder derivative is, undoubtedly, negative and therefore the maximum exists if condition $f^{\prime}=0$ is satisfied; this condition allows to determine the value of share $n$ for which the referred inflection point is accomplished. The respective result is $n^{\max }=\left(\varphi_{I}^{c}-\varphi_{E}^{c}\right) /\left(2 \varphi_{I}^{c}\right)$. The maximum change, found for $n^{\max }$, will be $\Delta n^{\max }=f\left(n^{\max }\right)=\left(\varphi_{I}^{c}+\varphi_{E}^{c}\right)^{2} /\left(4 \varphi_{I}^{c}\right)$; observe that $\Delta n^{\max }$ is necessarily above zero (the value of $\Delta n_{t}$ for $n_{t}=1$ ) and it is not an amount inferior to $\varphi_{E}^{c}$ (the value of $\Delta n_{t}$ for $\left.n_{t}=0\right)$.

The computed value $n^{\max }$ is a quantity below 1 , but it can be either positive or negative. If $\varphi_{I}^{c}>\varphi_{E}^{c}$, then $n^{\max }$ is a positive value and, thus, the inflection point will exist for an admissible value of share $n_{t}$ : the adoption curve will be convex in a first phase, it reaches a maximum at $n^{\max }$ and then it becomes concave as the convergence towards $n^{*}=1$ is completed. In the opposite case, $\varphi_{I}^{c}<\varphi_{E}^{c}$, the inflection point occurs for a non admissible $n_{t}$ value and, thus, the function $f\left(n_{t}\right)$ will be decreasing in all the extent of the interval $n_{t} \in[0,1]$, i.e., the adoption curve will be concave throughout the diffusion process from $n_{0}=0$ (or any other initial share value) to $n^{*}=1$.

The second part of the proposition states that even when $\varphi_{I}^{c}>\varphi_{E}^{c}$, the acceleration phase is subject to a constraint. In particular, the maximum point $n^{\max }$ is necessarily lower than 0.5 , because otherwise $\varphi_{E}^{c}$ would be a non positive value (to confirm this just solve $n^{\max }<0.5$, in order to obtain an universal condition)

The properties of the diffusion by contagion, referred to in proposition 5, can be illustrated graphically. Figure 1 presents two possibilities for the relation between $n_{t}$ and $\Delta n_{t}$; we depict cases $\varphi_{I}^{c}>\varphi_{E}^{c}$ and $\varphi_{I}^{c}<\varphi_{E}^{c}$ (in order to construct the graphics, panel $\mathbf{A}$ of the figure takes $\varphi_{I}^{c}=0.25$ and $\varphi_{E}^{c}=0.05$; panel $\mathbf{B}$ considers $\varphi_{I}^{c}=0.1$ and $\left.\varphi_{E}^{c}=0.15\right)$.

$$
\text { *** Fig. } 1^{* * *}
$$


Diffusion Dynamics in Economics: an Application to Fiscal Policy

In figure 1, panel $\mathbf{A}$ shows how $n_{t}$ varies with its own value for $\varphi_{I}^{c}>\varphi_{E}^{c}$. The change in $n_{t}$ is always positive and the system converges towards the stable point $n^{*}=1$. An s-shaped evolution for the variable under appreciation is confirmed by the hump-shaped form of the displayed relation. In a first stage, $\Delta n_{t}$ increases, then it reaches a maximum and afterwards it begins to fall, meaning that the relation of $n_{t}$ with time is such that the corresponding path is convex before point $n^{\max }$ is reached and then it becomes concave as the system converges to its resting point $n^{*}=1$.

The case $\varphi_{I}^{c}<\varphi_{E}^{c}$ is represented in panel $\mathbf{B}$ and, again, it indicates the presence of convergence to $n^{*}=1$, for a dynamic process that initiates in some admissible $n_{0}$ value. Once more, the contagion effect is stable in the sense that the epidemic will, for sure, spread throughout the entire population. However, in this second case, the maximum of the function does not fall in the interval of possible values of $n_{t}$. Therefore, $\Delta n_{t}$ decreases along the whole adjustment range. Hence, the s-shaped trajectory for $n_{t}$ will no longer be observed. This path will have a concave shape indicating that as $n_{t}$ increases the number of new adopters will be progressively smaller independently of the number of already existing adopters. The border case, where the rates of internal and external current non-adopters are identical is similar in nature with the one in panel $\mathbf{B}$, where now the maximum of the function is found at point $n_{t}=0$; in this case, $\Delta n^{\max }=\left(\varphi_{I}^{c}+\varphi_{E}^{c}\right)^{2} /\left(4 \varphi_{I}^{c}\right)=\varphi_{E}^{c}$. Once again, the adoption curve is strictly concave.

Let us now turn to the setting of diffusion under social influence. In this second scenario, people will adopt the new behavior / absorb the new information when a certain threshold of individuals has already changed its behavior or its degree of attentiveness relatively to the new piece of information.

Individual agents will possess different degrees of responsiveness to social influence (i.e., different adoption thresholds). A low threshold value signifies that agents adopt the innovation even if only a small share of individuals has already adopted; a high threshold will reflect a low responsiveness to the behavior of others. In this type of diffusion process, it is assumed that at a given time $t$ the proportion of individuals whose thresholds have been 
Diffusion Dynamics in Economics: an Application to Fiscal Policy

crossed is given by the cumulative distribution function of $n_{t}, F\left(n_{t}\right)$. Of this proportion, a subset $n_{t}$ of individuals have already adopted the innovation and, thus, one can express the share of individuals who have crossed the threshold but have not yet adopted by $F\left(n_{t}\right)-n_{t}$. We consider $\varphi^{s i}>0$ as the rate of conversion, and therefore the adoption process is presentable under the difference equation

$$
\Delta n_{t}=\varphi^{s i}\left[F\left(n_{t}\right)-n_{t}\right] \quad n_{0} \text { given }
$$

The specific shape of the diffusion process will depend on the type of distribution one considers, although one can recall some generic properties that help in understanding the main features of the underlying dynamics. A distribution function is, by definition, a non-decreasing function defined in the line of real numbers and with outcomes in the interval $[0,1]$. Moreover, the derivative $F^{\prime}$ corresponds to the respective probability density function.

The steady-state is now the point for which $F\left(n^{*}\right)=n^{*}$; this will not be necessarily accomplished since the cumulative distribution function of $n_{t}$ will exhibit a value that cannot be lower than $n_{t}$. In the examples to explore later, it is true that $F(1)$ is asymptotically equal to 1 , and therefore we can consider this to be an asymptotic steady-state; however, if $F\left(n^{*}\right)=n^{*}$ does not hold for $n_{t}=1$, it will not hold for any other, lower than 1 , value of the variable. Thus, we just remark that for $n_{t}=1, \Delta n_{t}=\varphi^{s i}[F(1)-1]$, which can be zero or a value slightly above zero. We can also compute the change in $n_{t}$ for $n_{t}=0$, which yields $\Delta n_{t}=\varphi^{s i} F(0)$. Finally, it is also a point of interest the one for which the right hand side of (14) reaches a maximum. This will occur, as in the contagion case if the mentioned function has a negative second order derivative and it is possible to find the point for which the first derivative is equal to zero. The first derivative is $\varphi^{s i}\left(F^{\prime}-1\right)$ and the second is $\varphi^{s i} F^{\prime \prime}$. Thus, if the derivative of the density function is a negative value, we find a maximum for $n_{t}$ at the point in which $F^{\prime}=1$, i.e., the density function is equal to 1 . The diffusion result is expressed in proposition 6 .

Proposition 6 Under social influence, the diffusion process is s-shaped for 
Diffusion Dynamics in Economics: an Application to Fiscal Policy

$F\left(n^{\max }\right)-F(0)>n^{\max }$, with $n^{\max }$ the solution of $F^{\prime}=1$.

Proof. Above, one has remarked that the eventual maximum value of $n_{t}$ is given by the solution of $F^{\prime}=1$. Once $n^{\max }$ is found, we observe that $\Delta n^{\max }=\varphi^{s i}\left[F\left(n^{\max }\right)-n^{\max }\right]$. We want to compare this value with the value of $\Delta n_{t}$ under $n_{t}=0$ and $n_{t}=1$. As remarked, the latter case is such that the change in the variable is zero or a value close to zero; thus, it remains to be compared the value of $\Delta n$ when $n_{t}=0$, with $n^{\max }$. If the maximum change lies to the right of $\varphi^{s i} F(0)$, we can guarantee, as in the contagion case, the presence of a maximum in the interval $n_{t} \in(0,1)$, which implies that the diffusion process will exhibit an inflection point, i.e., a point that guarantees the s-shape form for the trajectory of the share variable. The required condition is the one in the proposition

A common distribution that we can resort to is the normal distribution. Assume an average value $\mu \in(0,1)$, which represents the expected value of the threshold of adoption for any individual in the population and let $\sigma$ be the standard deviation of the same process. The corresponding density function is

$$
F^{\prime}=\frac{\exp \left[-\frac{\left(n_{t}-\mu\right)^{2}}{2 \sigma^{2}}\right]}{\sqrt{2 \pi \sigma^{2}}} .
$$

The derivative of this function is straightforward to compute:

$$
F^{\prime \prime}=-\frac{\left(n_{t}-\mu\right)}{\sigma^{2}} \frac{\exp \left[-\frac{\left(n_{t}-\mu\right)^{2}}{2 \sigma^{2}}\right]}{\sqrt{2 \pi \sigma^{2}}}
$$

This last expression will correspond to a negative value, for $n_{t}>\mu$. Therefore, we confirm the existence of a local maximum as long as the value of the variable is above the corresponding average. This maximum is the solution of $F^{\prime}=1$, which, in the normal distribution case, corresponds to $n=\mu \pm \sigma \sqrt{-\ln \left(2 \pi \sigma^{2}\right)}$. We have two solutions, but only one can correspond to a maximum given the condition $n_{t}>\mu$; thus, $n^{\max }=\mu+\sigma \sqrt{-\ln \left(2 \pi \sigma^{2}\right)}$. The diffusion process will be s-shaped (rather than concave throughout) if $n^{\max }>0$, a fact that is straightforward to observe.

Finally, one can look at an environment of social learning. This last 
Diffusion Dynamics in Economics: an Application to Fiscal Policy

setting does not consider that agents adopt a new information set, idea or behavior just because others have done so in the past (this was, in fact, what both the contagion and social influence scenarios implicitly meant). Alternatively, learning implies that agents will weight information about prior outcomes when deciding whether to adopt. Analytically, this translates into the replacement of the distribution function in equation (14) by the distribution of the sum of all shares $n_{s}$ from $s=0$ to $s=t$ (i.e., to the present moment). In the learning case, the relevant variable is the cumulative information generated by all prior adopters from the time they first adopted. Therefore, the dynamic equation translating the diffusion process will be

$$
\Delta n_{t}=\varphi^{s l}\left[F\left(\sum_{s=0}^{t} n_{s}\right)-n_{t}\right] \quad n_{0} \text { given }
$$

Again, the parameter in the equation, $\varphi^{s l}$, represents a positive rate of adoption.

The difference between social influence and social learning relates to what information is assumed relevant in order to formulate a decision. Under social influence, agents choose resorting to the most recent information. Considering social learning, all the payoffs in the past are taken as relevant information. A more realistic approach would be to assume that payoffs received in the past are less valuable in terms of current decisions. Letting $\rho \in(0,1)$, we could present a more sophisticated version of the social learning dynamics as

$$
\Delta n_{t}=\varphi^{s l}\left[F\left(\sum_{s=0}^{t} \rho^{t} n_{s}\right)-n_{t}\right] \quad n_{0} \text { given }
$$

Some basic properties of the social influence case also apply to social learning. More than in the previous case one must expect $F(1)$ to approach 1, and thus $n_{t}=1$ will be an asymptotic steady-state. Moreover, the condition underlying the existence of a maximum is of similar nature: $0<n^{\max }<1$ or, equivalently, $F(0)<F\left(m^{\max }\right)-n^{\max }<F(1)-1$, with $m_{t}:=\sum_{s=0}^{t} n_{s}$. A relevant result of the comparison between the social influence and the social learning cases is presented in proposition 7.

Proposition 7 Consider some cumulative distribution function $F\left(n_{t}\right)$. If 
Diffusion Dynamics in Economics: an Application to Fiscal Policy

under both social influence and social learning, the share variable follows an s-shaped evolution, then the inflection point is first crossed for social learning then for social influence.

Proof. The result in the proposition is intuitive because in the learning case the argument of the distribution function is a cumulative value, thus a quantity that is larger than the one under social influence. In reality, if $F^{\prime}\left[\left(n^{\max }\right)^{s i}\right]=1$ and $F^{\prime}\left[\left(m^{\max }\right)^{s l}\right]=1$, we must have $\left(n^{\max }\right)^{s i}=\left(m^{\max }\right)^{s l}$, which is equivalent to $\left(n^{\max }\right)^{s i}=\sum_{s=0}^{t-1} n_{s}+\left(n^{\max }\right)^{s l}$, i.e., we confirm that $\left(n^{\max }\right)^{s l}$ must be lower than $\left(n^{\max }\right)^{s i}$

We end this section with a numerical example that compares the three types of diffusion processes and that allows to illustrate the described generic results. We assume, for the social influence and for the social learning cases, a normal distribution with $\mu=0.1$ and $\sigma=0.1$. The adoption rate parameters will be $\varphi_{I}^{c}=\varphi^{s i}=\varphi^{s l}=0.25$ and $\varphi_{E}^{c}=0.05$. Similar adoption rates will allow us to compare outcomes. The results are displayed in figures 2 and 3 . Figure 2 presents the $\left(n_{t}, \Delta n_{t}\right)$ diagram for the three kinds of diffusion, while figure 3 displays the s-shaped trajectories of $n_{t}$.

$$
\text { *** Fig. 2,3*** }
$$

Generic results can be confirmed:

- In the three cases, we have an s-shaped diffusion process, where is visible the stable nature of the underlying dynamics. After more or less 20 to 25 time periods, the steady-state of full adoption is asymptotically accomplished. In figure 2 , we observe that the relation $\left(n_{t}, \Delta n_{t}\right)$ is hump-shaped for every possible form of diffusion, what gives place to the diffusion trajectories in figure 3 .

- Simple observation allows to perceive that (for the chosen parameter values) social influence and social learning generate relatively similar outcomes and that the contagion trajectory implies, relatively to the others, a slower adoption rate in a first phase and a recovery after some time periods (around 10). 
Diffusion Dynamics in Economics: an Application to Fiscal Policy

- For the contagion case, the maximum value of $\Delta n_{t}$ occurs at $n^{\max }=$ $\left(\varphi_{I}^{c}-\varphi_{E}^{c}\right) /\left(2 \varphi_{I}^{c}\right)=0.4$; this can be confirmed in figure 3 . The statements in proposition 5 are confirmed, i.e., the s-shape form of the adoption path is present because we have chosen values of parameters such that $\varphi_{I}^{c}>\varphi_{E}^{c}$ and the inflection point $n^{\max }$ lies below the $50 \%$ adoption rate.

- Under social influence, we find the maximum of $\Delta n_{t}$ by solving equation $F^{\prime}=1$, which, for the selected normal distribution allows for finding the solution $n^{\max }=0.2663$. Thus, $\Delta n_{t}=0.25(0.9519-0.2663)=$ 0.1714. Note that $n^{\max }$ is smaller in the social influence case then in the contagion setup; therefore, the time length in which $n_{t}$ varies at an increasing rate is larger in the contagion case than under social influence; nevertheless, this second type of diffusion will imply a faster initial increase of the share $n_{t}$. Proposition 6 is straightforward to apply to this simple example: we confirm that the diffusion process is s-shaped because $F\left(n^{\max }\right)-F(0)>n^{\max } \Leftrightarrow 0.9519-0.1587>0.2663$ $\Leftrightarrow 0.7932>0.2663$.

- In what concerns social learning, the first phase of convergence towards full adoption is relatively faster than under social influence. This is an expected result since, as referred, under learning information about past adoptions is also relevant (and not only the number of current adopters). Proposition 7 is also confirmed by noticing that the maximum $\Delta n_{t}$ is found, in the learning case, for $n^{\max }=0.2352$, a value that is clearly below the one found in the case of social influence.

\section{Results on Wealth, Time Allocation and Consumption}

Our main purpose is to evaluate the impact of fiscal policy changes over the steady-state values of wealth, time allocation and consumption, in the presence of the assumption on bounded rationality / behavior diffusion. The 
aggregate response to disturbances is sluggish because only a small share of agents instantly solve the new optimization problem (i.e., the problem involving the new tax rates), while all the other agents will also adopt the behavior compatible with the new fiscal conditions but in posterior time moments, following the diffusion pattern.

Several types of policies can be taken into consideration. For instance, we can distinguish between permanent and temporary policy changes. In the first case, given one of the three types of diffusion processes discussed in the previous section, there will be a gradual departure from the first steady-state set of values in the direction of the post-perturbation equilibrium, which will be accomplished for sure. If the policy change has a temporary nature (e.g., a six-period decrease in the labor income tax rate), some individuals will be able to accommodate their optimal behavior to the tax change, but others, that are slower to react, will continue with their pre-perturbation behavior and when the tax reversal takes place they will not have to change their behavior back. The initial steady-state is recovered as the agents with the faster adoption capacity adjust back to the original tax rate.

The exact mechanism through which the diffusion after a policy shock functions will be the following:

1. The level of wealth is a state variable; as a result, agents in the economy cannot manipulate the value of this variable. If a tax change occurs, this will instantly modify the endowment of wealth held on the aggregate by the economy. The steady-state level of wealth is given in expression (9); according to this expression, the only change in a tax rate that modifies the long-run level of wealth is the change in the savings tax rate. Following proposition 1 , an increase in $\tau_{s}$ will lead to a fall in $a^{*}$. Independently of rationality issues involving the behavior of economic agents, the value of this state variable will always suffer a full instantaneous impact that occurs when a fiscal policy change is triggered. Any change in the tax rates $\tau_{\ell}$ and $\tau_{c}$ leave the trajectory of $a^{*}$ unchanged.

2. Sluggishness will basically be the result of the incapacity most agents exhibit in perceiving instantly which is their best decision in terms of laborleisure choices. As referred, the capacity to immediately re-optimize is re- 
Diffusion Dynamics in Economics: an Application to Fiscal Policy

stricted to a small share of agents; all the others will just follow the pioneer behavior of the 'innovators' and adopt, sooner or later, a similar kind of labor participation behavior. Thus, when a policy change occurs, agents will gradually (and not instantly) switch from the level of participation in the labor market that allowed to accomplish the pre-shock optimal utility outcome to the labor-leisure option that maximizes the post-perturbation utility level. Recall that the steady-state optimal labor share is given by expression (10). From this expression, one has realized that $\tau_{c}$ does not exert any influence over the steady-state time allocation choice; a change in $\tau_{s}$ induces a variation of the same sign in $\ell^{*}$, while $\Delta \tau_{\ell}$ will lead to a change of the same sign in the leisure share (i.e., a variation of opposite sign in $\ell^{*}$ ).

While in section 3 we have computed the long-term impact of the policy changes over the allocation of the agents' time, now we are interested in the short-run. The short-run should be interpreted as the time span along which the diffusion process is taking place; therefore, the long-run will be the setting in which the diffusion process is asymptotically terminated. Thus, when a tax change $\Delta \tau$ occurs, this will be perceived, in terms of labor market decisions, only by a share $n_{t}$ of individuals; all the others will continue acting as if the policy change had not occurred. On the aggregate, when the policy shock takes place, the relevant tax rate involving time allocation decisions will be $\tau^{\prime}=\tau+n_{t} \Delta \tau$. I.e., at time $t$, a fraction $n_{t}$ of the population has already brought the new tax rate $\left(\tau^{\prime}\right)$ into their decision problem, and a fraction $1-n_{t}$ continues to consider as relevant the original tax rate $\tau$. Note that $n_{t} \Delta \tau$ will coincide with $\Delta \tau$ after some time periods have passed - in the examples of the previous section, around 20 to 25 periods - but there is a transition phase in which the full extent of the tax change is not included in agents' decisions and therefore these depart from the optimal outcome).

3. The other control variable of the problem is consumption. If agents are unable to instantly adapt their time allocation behavior, this inertia will have to be accommodated in terms of consumption levels. If agents work more or less than what is optimal at a given time, this implies that consumption will have to be changed accordingly. From the steady-state conditions we can 
Diffusion Dynamics in Economics: an Application to Fiscal Policy

recover the following expression:

$$
c^{*}=\frac{1}{1+\tau_{c}}\left[\left(1-\tau_{s}\right) r\left(a^{*}\right)^{\alpha}+\left(1-\tau_{\ell}\right) w \ell^{*}\right]
$$

This relation indicates that when a fiscal policy measure is adopted and this implies that $\ell^{*}$ does not vary in the exact amount as it should, this non optimal variation must be compensated by consumption, i.e., the consumption level will also change in an amount that differs from the optimal one. Note that these observations make sense only for $\tau_{s}$ and $\tau_{\ell}$; tax rate $\tau_{c}$ does not exert any effect over labor-leisure choices and, consequently, consumption can move instantly to the new equilibrium when a change in the consumption tax rate takes place.

The main conclusion to draw is that as long as $n_{t}<1$, a change in $\tau_{s}$ or $\tau_{\ell}$ will imply that the steady-state levels of time allocation and consumption will depart from the corresponding levels that would maximize utility. Thus, the consequence of the assumed kind of bounded rationality will be a loss of long-term welfare, because agents are unable to instantly adapt to new fiscal conditions (this will become clear with the analysis in the following section).

General results are hard to evaluate and typically will not produce informative outcomes. Thus, for an overall assessment of the implications of policy changes, we will consider a numerical example. We will work with the diffusion processes characterized in section 4 and displayed in figures 2 and 3 . Furthermore, we recover the array of parameter values used in section 3, i.e., $m=0.2, w=0.25, r=0.1, \alpha=0.25$ and $\beta=0.96$. Finally, we need initial values for the tax rates; let $\tau_{s}=0.25$ and $\tau_{\ell}=\tau_{c}=0.2$. The assumed parameter values allow for a straightforward computation of steady-state optimal levels of wealth, time allocation and consumption: $a^{*}=0.3448, \ell^{*}=0.7854$ and $c^{*}=0.1788$. The computed time allocation value indicates that in this economy, under the specified settings, around $78.54 \%$ of the available time of the agents is, in the steady-state, allocated to labor hours.

We will consider the following alternative policies:

1. A permanent change in the tax rate on financial income: $\Delta \tau_{s}=0.05$; 
Diffusion Dynamics in Economics: an Application to Fiscal Policy

2. A permanent change in the labor income tax rate: $\Delta \tau_{\ell}=0.03$;

3. A temporary change ( 6 periods) in the labor income tax rate: $\Delta \tau_{\ell}=$ -0.05 ;

4. A temporary change (6 periods) in the tax rate over consumption: $\Delta \tau_{c}=0.02$.

Many other policy measures could be considered, including fiscal plans combining the simultaneous manipulation of several tax rates. In order to illustrate our arguments, the considered changes are enough.

Consider first the policy measure $\Delta \tau_{s}=0.05$. According to proposition 1, this will imply a fall in the level of wealth and in consumption and a reallocation of time in favor of labor hours, if the agents want to continue to maximize utility. The long-run effects are the following: $\Delta a^{*}=-0.0303$, $\Delta \ell^{*}=0.0042$ and $\Delta c^{*}=-0.0035$. The short-run effects will correspond to the evolution implied by the diffusion process. These effects are depicted in figures 4,5 and 6 . The policy change takes place, in the graphics, at time $t=10$.

$$
\text { *** Fig. 4, 5, 6*** }
$$

The proposed mechanism implies an immediate change in the value of the state variable as the result of the fiscal policy change. In what concerns the control variables, there is a convergence towards the new equilibrium. When the tax rate change takes place, households will start modifying their behavior concerning time allocation at a gradual pace. There is a diffusion process that implies an s-shaped trajectory from the first to the second equilibrium time allocation point, which is accomplished, more or less, in 20 to 25 time periods (see figure 5). Relatively to consumption, this has to adjust following rule (17). Figure 6 shows that, with the policy shock, consumption will immediately fall, but because the labor participation does not increase as much as it should in the short-run in order to achieve an optimum, the fall in consumption will initially be stronger than the one observed in the long-run. 
Diffusion Dynamics in Economics: an Application to Fiscal Policy

Through figures 4 to 6 , besides verifying the evidence on the sluggish adjustment of control variables, we also confirm the different patterns of adjustment for different kinds of diffusion processes: social learning provides the fastest adjustment to the new equilibrium position and contagion the slowest.

Next, assume the permanent positive change in the labor income tax rate. Proposition 2 has indicated that this type of policy measure does not have an impact on wealth (this remains at level $a^{*}=0.3448$ ); with respect to the other variables, one should expect a fall in the share of time allocated to labor and also a decrease in consumption. Nevertheless, these are long-term effects that overlook the transitional dynamics phase implied by diffusion. The relevant pictures are now figures 7 and 8 .

$$
\text { *** Fig. } 7,8^{* * *}
$$

These figures confirm the fall in the values of $\ell^{*}$ and $c^{*}$ as the result of taking $\Delta \tau_{\ell}=0.03$. The changes in the steady-state values are, respectively, $\Delta \ell^{*}=-0.0019$ and $\Delta c^{*}=-0.0052$. In the short-run, the labor share will gradually fall to the new steady-state, while consumption decreases, however initially in an extent that is inferior to the long-term change. The differences in the followed trajectories implied by the three different types of diffusion are not too significant, although they are exactly ranked as in the previously illustrated case: social learning provides the fastest convergence towards the long-run locus, followed by the social influence pattern of diffusion, and finally contagion.

Now, consider the temporary policy consisting in a six period fall in the labor income tax. A negative change in $\tau_{\ell}$ allows for an increase in the steadystate values of $\ell_{t}$ and $c_{t}$, but now the change in the tax rate is temporary - it will be maintained only for 6 time periods. Thus, the new equilibrium $\ell^{*}=0.7883$ and $c^{*}=0.1875$ will never, in fact, be accomplished. There is an inertial convergence to this new equilibrium point following the diffusion process, that is interrupted at $t=16$ (it was initiated at $t=10$ ). When the initial fiscal policy is recovered, a new diffusion process is initiated back to the original steady-state. See figures 9 and 10. 
Diffusion Dynamics in Economics: an Application to Fiscal Policy

$$
\text { *** Fig. 9, } 10^{* * *}
$$

Again, it is social learning that provides a faster initial adjustment to the shock (and also the fastest change back to the original state). Notice that in the case of consumption, the initial change leads to a positive jump and then to a phase of slow positive adjustment towards the new equilibrium that is interrupted when the tax rate changes back to its initial value. The recovery of the initial tax rate produces an immediate jump to a value that is close to the initial steady-state, but a small adjustment process is yet required for the original consumption state to be fully recovered.

Finally, we consider another temporary policy respecting to a change in the consumption tax rate. Proposition 3 states that this tax rate has effect solely over consumption, leaving unchanged the level of wealth and the distribution of time between labor and leisure. The assumption underlying diffusion according to which this will occur through a perturbation over the optimal decisions concerning time allocation implies that this kind of policy will not exert any short-run effect. Thus, time trajectories of $a^{*}$ and $\ell^{*}$ are left unchanged, and consumption will be, in a six period time length, lower than the one implied by a tax rate $\tau_{c}=0.2$; i.e., $c^{*}=0.1788$ for $t=1, . ., 10 ; 17, \ldots$; and $c^{*}=0.1756$ for $t=11, \ldots, 16$.

\section{Welfare Effects}

If agents are boundedly rational and, as a result, display distinct velocities of reaction when faced with a policy change that has implications over their optimal problem, there is a sluggish adjustment of macro control variables towards a new steady-state, after the policy disturbance takes place. The most relevant consequence of the characterized framework is that we will be faced with temporary departures from maximum utility levels, independently of the nature of the policy (i.e., whether it is temporary or permanent). Longrun utility has been presented in equation (12) and, according to proposition 4 , typically utility falls with a tax increase (the only exception occurs for the labor income tax rate under very strict circumstances). 
Diffusion Dynamics in Economics: an Application to Fiscal Policy

In this section, we briefly look at the departures from utility maximization given the bounded rational behavior that does not allow for an instant reoptimization.

Take, first, the permanent increase on the savings tax rate. Figure 11 displays, for the three types of diffusion processes, the difference between $U\left(c^{*}, 1-\ell^{*}\right)$ and the value of utility implied by the observed values of consumption and time allocation under diffusion. Note that for $t=1, . ., 10$, the long-term utility level is the one involving the original tax rate value, while after $t=10$ the values of $c^{*}$ and $\ell^{*}$ take into consideration the new tax rate.

$$
\text { *** Fig. } 11^{* * *}
$$

Although we have found a sluggish adjustment on labor participation following the fiscal shock, we observe an instantaneous departure from the utility level relatively to the benchmark optimal value (note that we are measuring deviations from the optimum, and thus this jump must be interpreted as a loss of utility); afterwards, a sluggish adjustment towards the maximum utility takes place. The optimal outcome is recovered once the diffusion process has ended (20 to 25 periods). Observe that contagion produces the largest departures relatively to the optimal result of utility maximization; at this level, social learning provides the less penalizing outcome.

The deviation relatively to the maximum utility can also be illustrated for policies $\Delta \tau_{\ell}=0.03$ (permanent) and $\Delta \tau_{\ell}=-0.05$ (temporary); we have seen that for $\Delta \tau_{c}=0.02$ there is no deviation relatively to the optimal outcome. Whether the tax rate on labor income falls or goes up, the sluggish adjustment leads to a less then optimal result. This is depicted in figures 12 and 13. The first of these figures presents a pattern of deviation from maximum utility very similar to the one in figure 11 (in both cases, fiscal policy measures are of a permanent nature). In figure 13, we have the case of a temporary policy; after the initial change, a relatively large deviation from the optimum is evidenced and, as time goes by, this is attenuated. Six periods later, a new shock occurs, that is, the tax rate changes back to its initial level. As a result, we have a new diffusion process and a new phase of departure relatively to the values of variables that maximize utility. The 
Diffusion Dynamics in Economics: an Application to Fiscal Policy

second deviation is not as strong as the first because at period 6 not all the agents would have already changed into the optimal behavior imposed by the new tax rate (in the second case there will be less agents adjusting their behavior).

$$
\text { *** Fig. 12, } 13 \text { *** }
$$

\section{Conclusion}

We have analyzed a standard intertemporal utility maximization model, in a setting where agents make choices concerning the allocation of time and about how much to consume of a given homogeneous good. The only departure from a fully competitive environment involving full rationality relates to the different capacities agents possess in what respects their ability to react to external events (these are basically fiscal policy changes, i.e., tax rate changes). Some agents will eventually have the capacity to re-compute a new optimal set of choices whenever this becomes necessary, but the full majority of the individuals in the population will not 'innovate' at this level; they will 'imitate' the behavior of the pioneer group. Heterogeneity arises because individuals will require different levels of adoption in order to decide whether to adopt themselves.

In our framework, the sluggishness or inertia in perceiving or accepting that economic conditions have changed after a fiscal policy disturbance is felt at the level of labor supply. As agents understand that the new tax rate is no longer compatible with the former optimum, they will gradually change their time allocation choices, following a diffusion process. Given some steady-state endowment of wealth, the departure from an optimal laborleisure choice has impact over consumption, which is also pushed away from the optimal outcome.

We have considered three forms of diffusion. We can conceive a scenario of pure contagion, where the simple presence in the same geographical space make agents follow, sooner or later, some rule adopted by others; we can also take social influence, a setting where agents adopt the same choices as 
others because they observe that the behavior is being adopted; and social learning, case in which all the past history concerning adoption is taken into consideration by the individual when deciding about the change in behavior (or about incorporating some set of new information). The developed exercise has indicated that social learning is the more effective type of diffusion process in allowing for a fast aggregate transition from the pre-shock to the post-perturbation equilibrium.

Under diffusion, fiscal policy disturbances have the following specific effects:

1. A positive change in the tax rate on financial wealth earnings leads to an instantaneous fall in the level of wealth, to a modification of laborleisure choices in favour of labor (this occurs under an s-shaped pattern of evolution over time of the labor share variable) and to a strong fall in consumption (larger than the long-run change) which is followed by a slight increase over time in the direction of the new steady-state consumption level.

2. A positive change in the labor income tax rate does not disturb the level of wealth and leads to an increase in leisure time (relatively to labor time) that occurs, again, under an s-shaped evolution. The aggregate consumption level will instantly decline, in the moment of the policy shock, but for a value slightly above the new steady-state one (the diffusion process will then generate the required adjustment to the longrun outcome).

3. A positive change on the tax rate over consumption does not influence wealth or time allocation decisions. Thus, consumption suffers, immediately, a re-adjustment to the new optimal value.

If the policy measures imply a decline rather than an increase on the tax rates, the effects will be symmetrical to the ones mentioned. Tax changes can also be temporary, and in this case two diffusion processes will occur; the second starts when the tax rate changes back to its initial value and ends when the original optimal point is recovered. 
Diffusion Dynamics in Economics: an Application to Fiscal Policy

The main consequence of the sluggish reaction of time allocation decisions to tax changes is that there will be a period of less than optimal decisions that produce an aggregate welfare loss in the sense that the level of utility will depart from its maximum value. If fiscal policy is frequently changed, this can be translated in persistent aggregate deviations from the optimal consumption and time allocation decisions. Thus, fiscal stability tends to be welfare enhancing.

\section{References}

[1] Akerlof, G. and J. Yellen (1985). "Can Small Deviations from Rationality Make Significant Differences to Economic Equilibria?" American Economic Review, vol. 75, pp. 708-720.

[2] Bass, F. (1969). "A New Product Growth Model for Consumer Durables." Management Science, vol. 15, pp. 215-227.

[3] Bass, F. (1980). "The Relationship between Diffusion Rates, Experience Curves and Demand Elasticities for Consumer Durables and Technological Innovations." Journal of Business, vol. 53, pp. 551-567.

[4] Beeby, M.; S. G. Hall and B. Henry (2001). "Rational Expectations and Near Rational Alternatives: How Best to Form Expectations." European Central Bank working paper series 086.

[5] Bomfim, A. and F. Diebold (1997). "Bounded Rationality and Strategic Complementarity in a Macroeconomic Model." Economic Journal, vol. 107, pp. 1358-1374.

[6] Gomes, O.; D. A. Mendes and V. M. Mendes (2008). "Bounded Rational Expectations and the Stability of Interest Rate Policy." Physica A, vol. 387, pp. 3882-3890. 
[7] Haltiwanger, J. and M. Waldman (1989). "Limited Rationality and Strategic Complements: the Implications for Macroeconomics." Quarterly Journal of Economics, vol. 104, pp. 463-483.

[8] Weder, M. (2004). "Near-rational Expectations in Animal Spirits Models of Aggregate Fluctuations." Economic Modelling, vol. 21, pp. 249-265.

[9] Young, H. P. (2009). "Innovation Diffusion in Heterogeneous Populations: Contagion, Social Influence, and Social Learning." American Economic Review, vol. 99, pp. 1899-1924. 


\section{Figures}
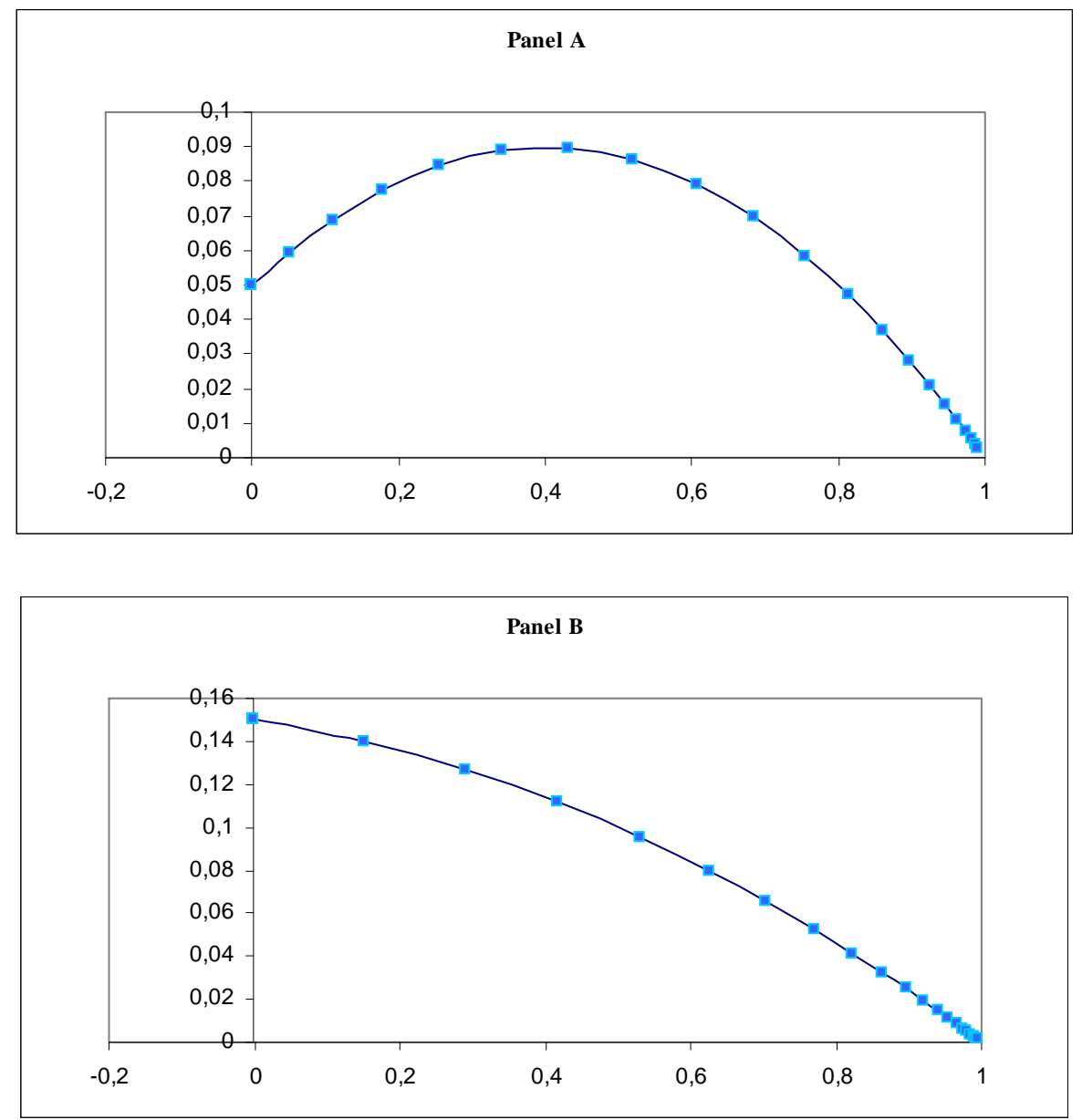

Figure 1 - $\left(n_{t}, \Delta n_{t}\right)$ diagram in the contagion diffusion case. Values of parameters: $\varphi_{I}^{c}=0.25, \varphi_{E}^{c}=0.05$ (panel A) $; \varphi_{I}^{c}=0.1, \varphi_{E}^{c}=0.15$ (panel B). 


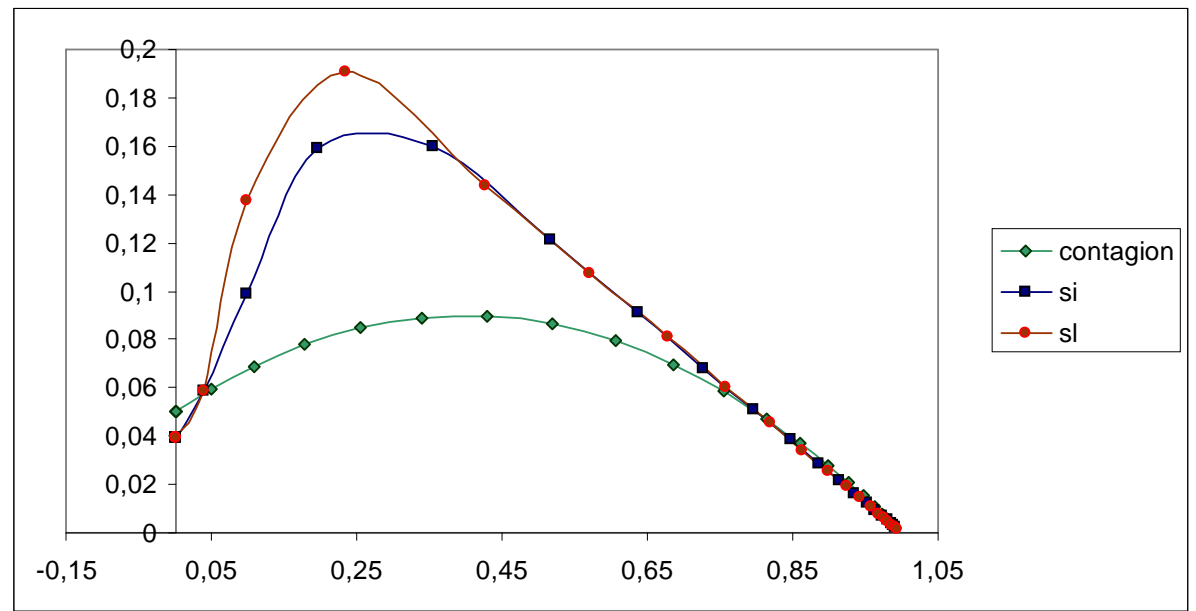

Figure 2 - $\left(n_{t}, \Delta n_{t}\right)$ diagram with diffusion under contagion, social influence and social learning.

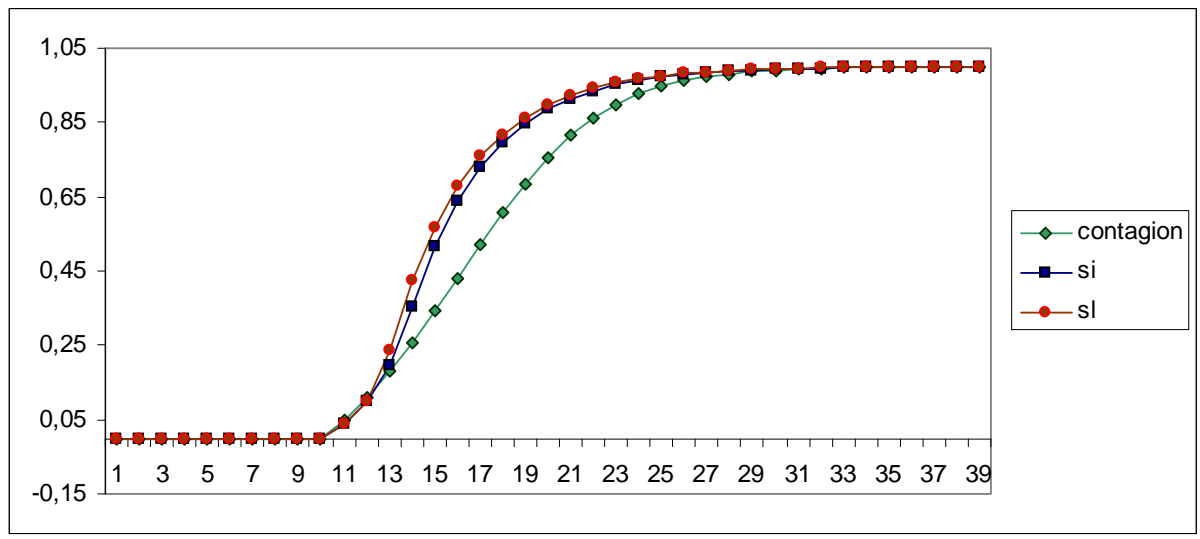

Figure 3 - Diffusion trajectories under contagion, social influence and social learning. 
Diffusion Dynamics in Economics: an Application to Fiscal Policy

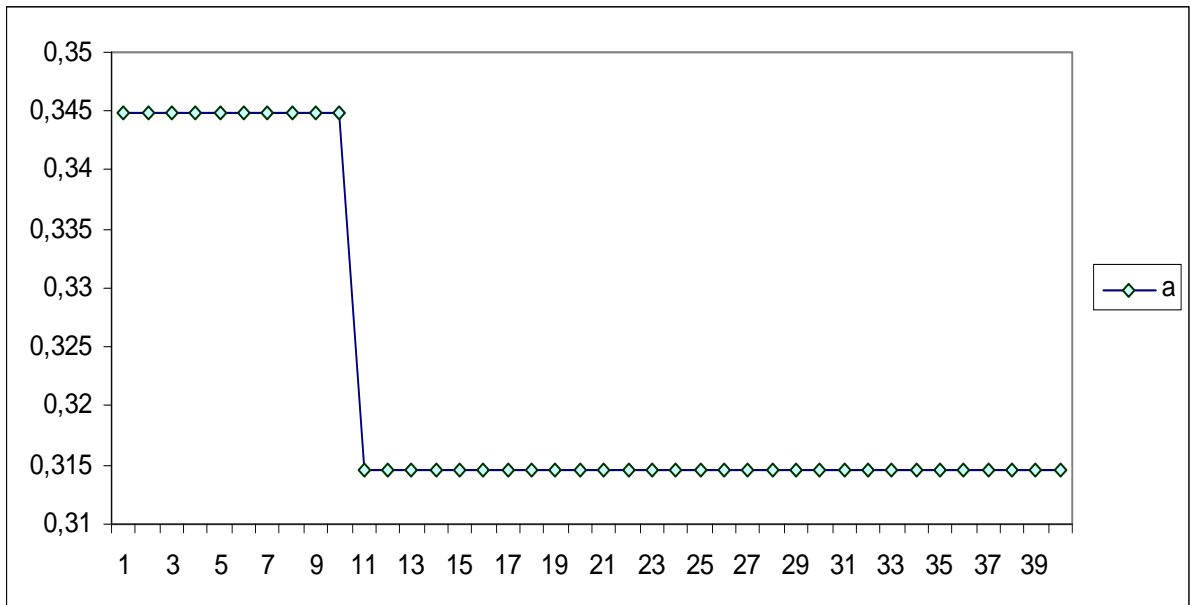

Figure 4 - A permanent change in $\tau_{s}$. Impact on the trajectory of wealth.

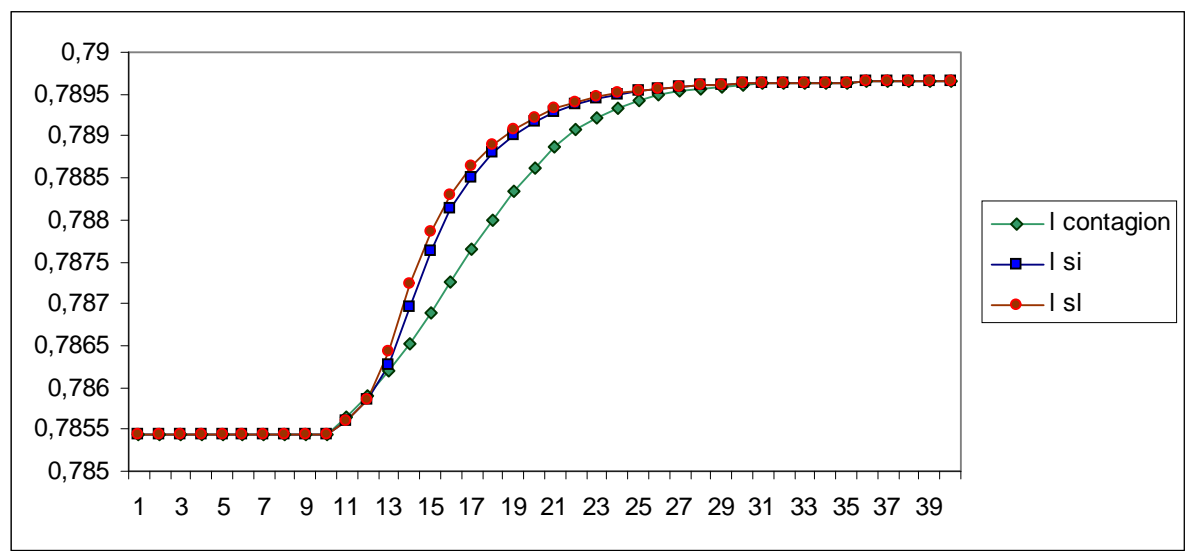

Figure 5 - A permanent change in $\tau_{s}$. Impact on the trajectory of labor time allocation. 
Diffusion Dynamics in Economics: an Application to Fiscal Policy

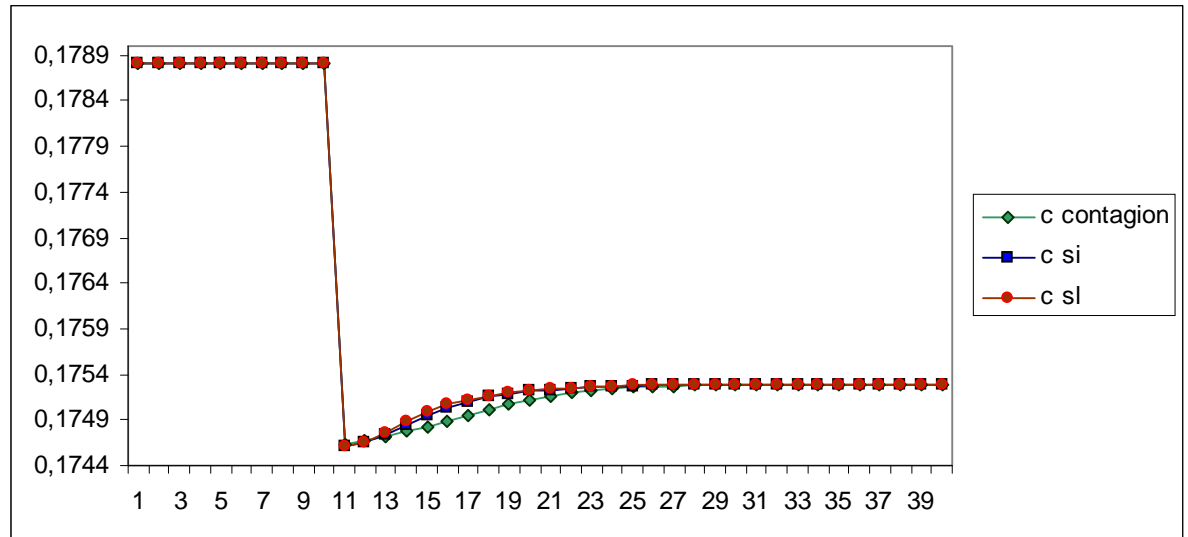

Figure 6 - A permanent change in $\tau_{s}$. Impact on the trajectory of consumption.

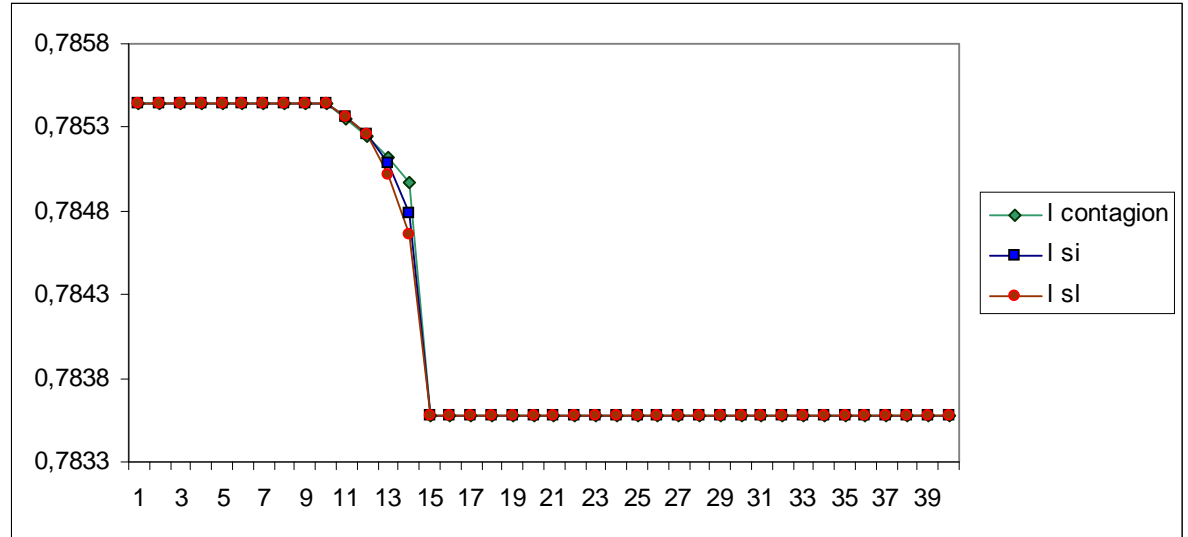

Figure 7 - A permanent change in $\tau_{\ell}$. Impact on the trajectory of labor time allocation. 
Diffusion Dynamics in Economics: an Application to Fiscal Policy

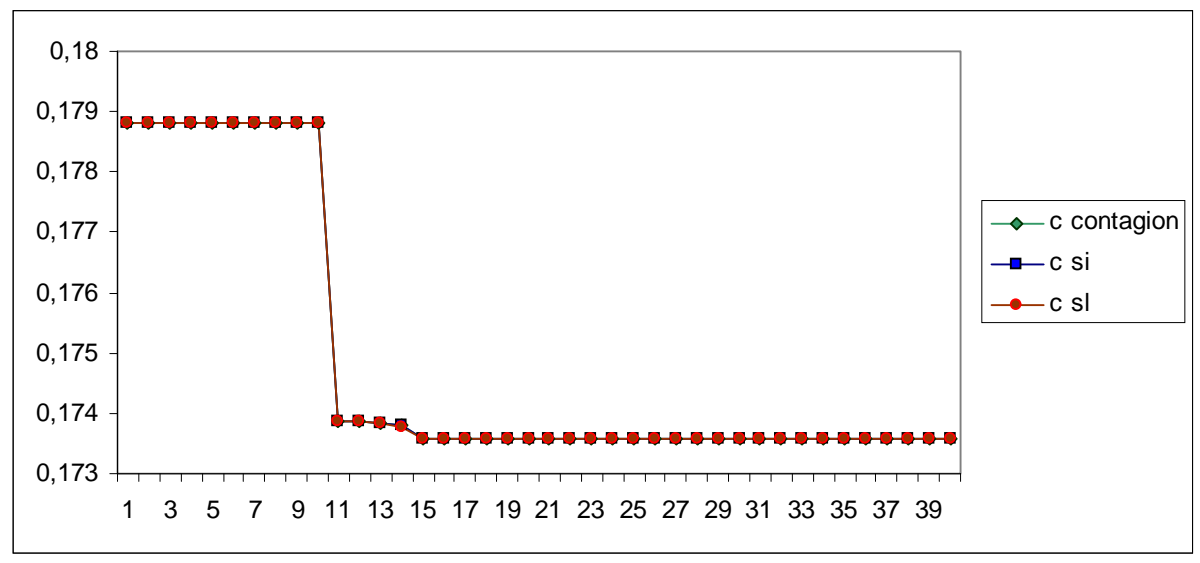

Figure 8 - A permanent change in $\tau_{\ell}$. Impact on the trajectory of consumption.

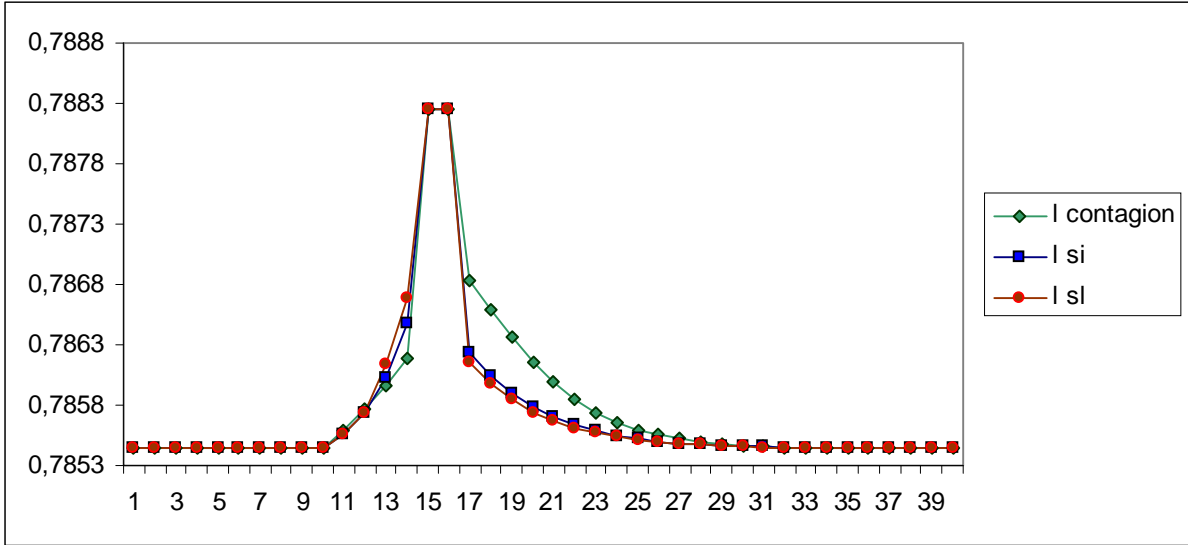

Figure 9 - A temporary change in $\tau_{\ell}$. Impact on the trajectory of labor time allocation. 
Diffusion Dynamics in Economics: an Application to Fiscal Policy

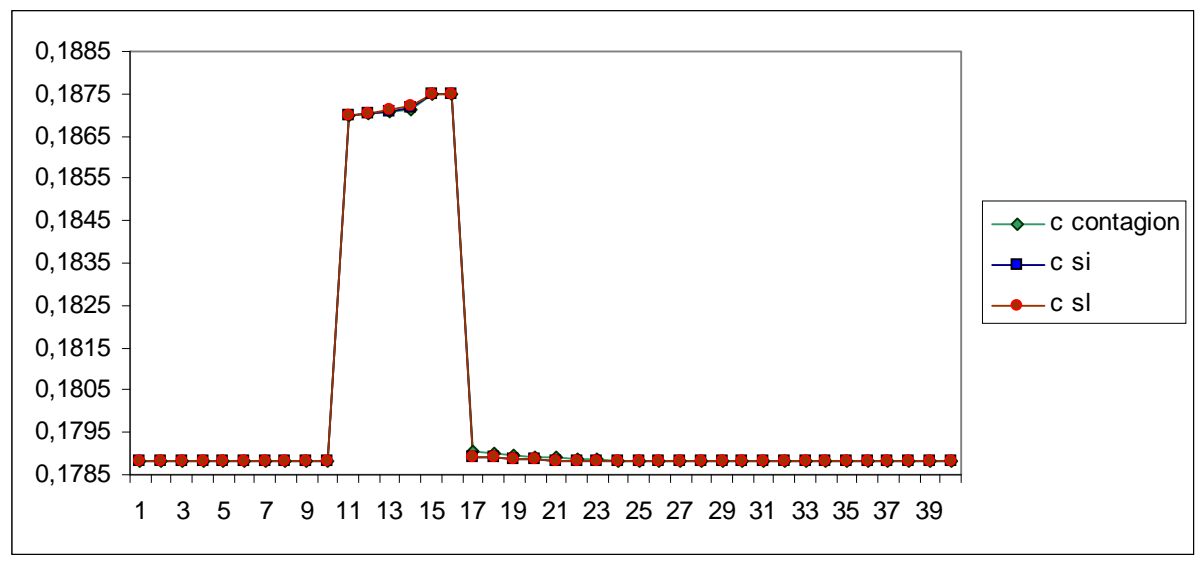

Figure 10 - A temporary change in $\tau_{\ell}$. Impact on the trajectory of consumption.

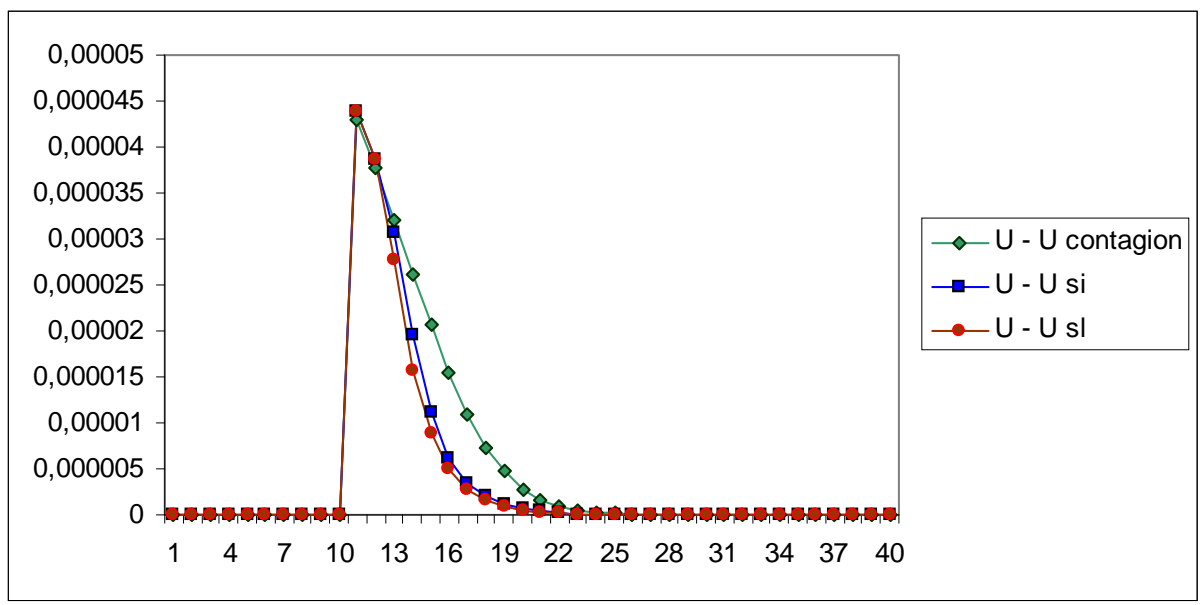

Figure 11 - Deviation between maximum utility and observed utility, for a permanent change in $\tau_{s}$. 
Diffusion Dynamics in Economics: an Application to Fiscal Policy

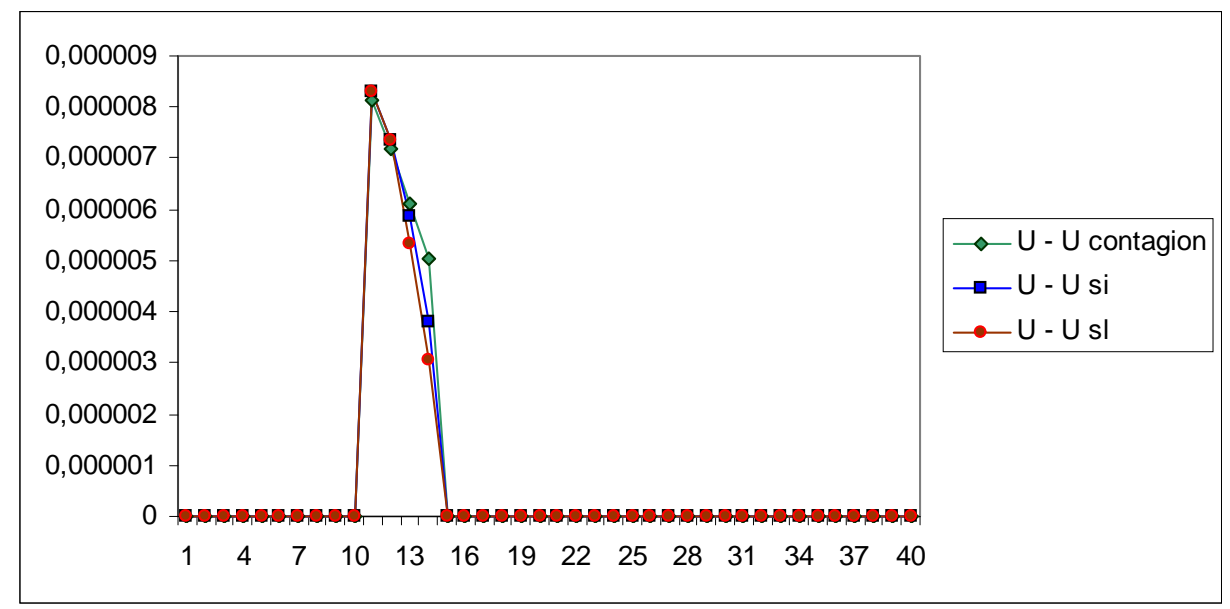

Figure 12 - Deviation between maximum utility and observed utility, for a permanent change in $\tau_{\ell}$.

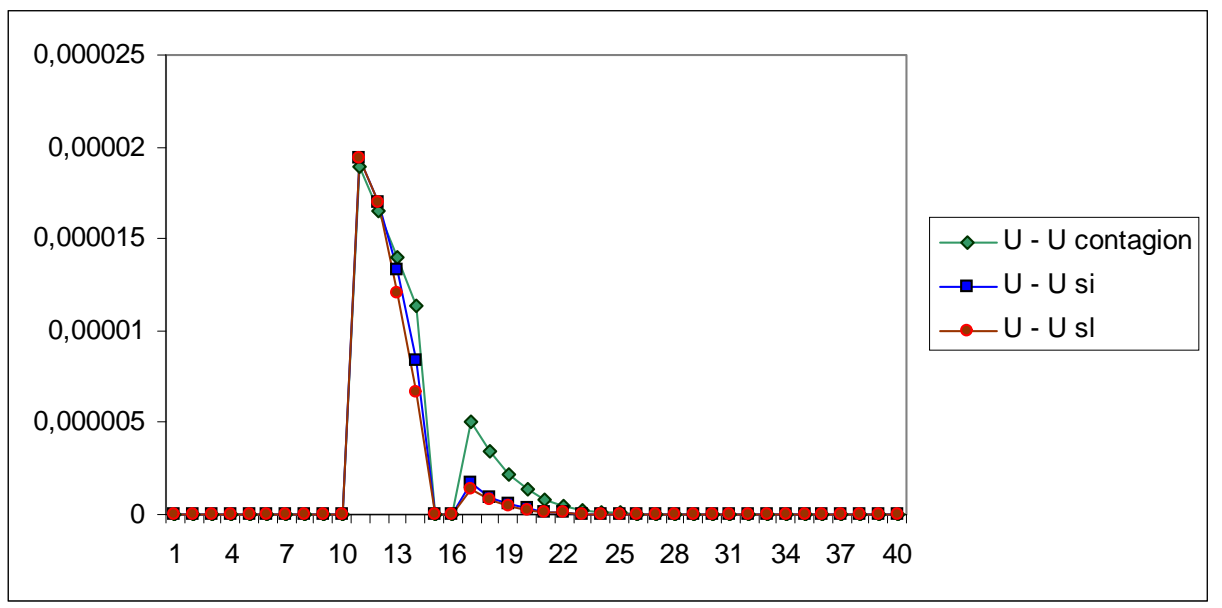

Figure 13 - Deviation between maximum utility and observed utility, for a temporary change in $\tau_{\ell}$. 